Gynäkologe 2012 · 45:94-94

DOI 10.1007/s00129-011-2876-6

(c) Springer-Verlag 2012

G, Emons ${ }^{1} \cdot$ K. Friese ${ }^{2} \cdot$ W. Distler ${ }^{3}$

${ }^{1}$ Universitätsfrauenklinik Göttingen

${ }^{2}$ Klinikum Innenstadt - Frauenklinik, LMU München,

${ }^{3}$ Klinik und Poliklinik für Frauenheilkunde und Geburtshilfe, Universitätsklinikum Dresden

\title{
Internistische Erkrankungen in der Schwangerschaft
}

von geburtshilflich interessierten Kardiologen, Nephrologen, Diabetologen, Hämatologen usw. ist es in vielen Fällen möglich, Hochrisikoschwangerschaften zu einem guten Abschluss zu bringen.

Voraussetzung für die zeit- und sachgerechte Einbindung von internistischen Kollegen sind allerdings gewisse internistische Grundkenntnisse beim Geburtshelfer nicht nur im Perinatalzentrum, sondern auch in der frauenärztlichen Praxis. Nur wenn potenzielle Probleme frühzeitig erkannt werden, können sie effektiv interdisziplinär gelöst werden. Frauenärzte, die junge Frauen betreuen, müssen deshalb heute wesentlich intensiver internistisch ausgebildet sein als noch vor 20 Jahren.

Mit dem vorliegenden Heft versuchen wir mit den Autoren Ihnen eine Auffrischung des Wissens über internistische Erkrankungen in der Schwangerschaft zu ermöglichen.

Adam et al. beschreiben die wichtigsten hämatologischen Erkrankungen in der Schwangerschaft und arbeiten heraus, dass nicht jede Thrombozytopenie Anlass für Notfallmaßnahmen ist.

Knabl et al. stellen das aktuelle Management von Diabetes (S3-Leitlinie) und Schilddrüsenerkrankungen in der Gravidität dar.

Hawighorst et al. erläutern die Führung von herzkranken Frauen in der Schwangerschaft und beleuchten das noch weitgehend unbekannte, aber sehr heimtückische Krankheitsbild der schwangerschaftsinduzierten Kardiomyopathie.

Aschka et al. skizzieren die wichtigsten Lebererkrankungen, die für die Schwangerschaft relevant sind, insbesondere auch die Störungen im Gallensäurestoffwechsel, die für die werdende Mutter lästig bis unerträglich, für den Feten bzw. das Neuge- borene jedoch lebensgefährlich sein können.

Zastrow $u$. Wirth liefern einen Überblick über Erkrankungen der Niere und der ableitenden Harnwege in der Gravidität.

Wir haben uns bemüht, die Artikel interdisziplinär erstellen zu lassen, um auf diese Weise internistische Kompetenz mit geburtshilflicher Relevanz zu verbinden. Wir sind uns bewusst, dass hier nur ein kleiner Teil von dem besprochen werden kann, was eigentlich unser internistisches Wissen sein sollte. Immerhin haben wir einen Anfang gemacht. Wenn Ihnen die Artikel zusagen, werden wir gerne ein weiteres Heft mit diesem thematischen Schwerpunkt auf den Weg bringen.

Viel Spaß beim Lesen wünschen

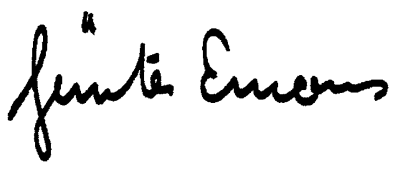

Prof. Dr. Günter Emons

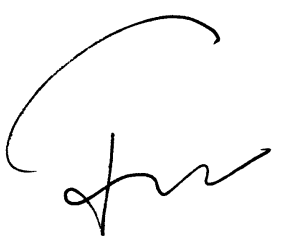

Prof. Dr. Klaus Friese

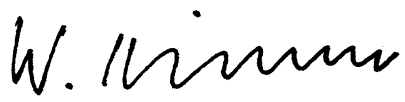

Prof. Dr. Wolfgang Distler

\section{Korrespondenzadresse}

\section{Prof. Dr. G, Emons}

Universitätsfrauenklinik Göttingen

Robert-Koch-Str. 40, 37075 Göttingen

emons@med.uni-goettingen.de 